\title{
Identification of the Dynamics of Biofouled Underwater Gliders
}

\author{
Enrico Anderlini and Giles Thomas \\ Department of Mechanical Engineering \\ University College London \\ London, UK \\ E.Anderlini@ucl.ac.uk
}

\author{
Stephen C.A. Woodward \\ Marine Autonomous and Robotic Systems \\ National Oceanography Centre \\ Southampton, UK
}

\author{
Daniel A. Real-Arce, Tania Morales, Carlos Barrera and J. J. Hernández-Brito \\ Oceanic Platform of the Canary Islands \\ PLOCAN \\ Las Palmas, Spain
}

\begin{abstract}
Marine growth has been observed to cause a drop in the horizontal and vertical velocities of underwater gliders, thus making them unresponsive and needing immediate recovery. Currently, no strategies exist to correctly identify the onset of marine growth for gliders and only limited datasets of biofouled hulls exist. Here, a field test has been run to investigate the impact of marine growth on the dynamics of underwater gliders. A Slocum glider was deployed first for eight days with drag stimulators to simulate severe biofouling; then the vehicle was redeployed with no additions to the hull for a further 20 days. The biofouling caused a speed reduction due to a significant increase in drag. Additionally, the lower speed causes the steadystate flight stage to last longer and thus a shortening of mission duration. As actual biofouling due to $p$. pollicipes happened during the deployment, it was possible to develop and test a system that successfully detects and identifies high levels of marine growth on the glider using steady-state flight data. The system will greatly help pilots re-plan missions to safely recover the vehicle if significant biofouling is detected.
\end{abstract}

Index Terms - underwater glider, biofouling, marine growth, system identification

\section{INTRODUCTION}

Underwater gliders (UGs) are a type of autonomous underwater vehicle that are being used extensively for long-term observation of key physical oceanographic parameters [1]. The vehicles profile vertically through the water by changing their buoyancy through a variable buoyancy device (VBD). Wings generate a forward motion component from the vertical motion. Their simple propulsion system, which consists of the VBD, pitch control and either roll control or a rudder, is highly efficient. Therefore, although they operate at low velocities $(\approx 0.3 \mathrm{~m} / \mathrm{s})$, the deployments of UGs can last for several months.

The long deployment duration in addition to regular surfacing to send data and receive new commands by satellite can result in significant marine growth problems [2]. This issue is particular pronounced in shallow, warm, tropical waters.

The research leading to these results has received funding from the European Unions Horizon 2020 research and innovation programme under grant agreement No 731103 .
Marine growth results in an increase in the weight of the glider and a significant drop in speed. Therefore, biofouling can represent a severe operational problem, with the vehicle possibly needing premature retrieval at sea.

To date, there are no systems to detect and track marine growth in real time. The study by Haldeman, et al. [2] investigated the impact of biofouling during a deployment in the South Atlantic a posteriori. In [3], marine growth was observed to cause a drop in speed with time and an increase of $1^{\circ}$ in the angle of attack over a whole deployment for the same pitch command. Additionally, medium to severe biofouling levels can cause a decrease in the lift coefficient of up to $40 \%$ and an increase in the drag coefficient of $90 \%$ [4].

This article describes field tests run as part of a EU Marine Robots Transnational Access (TNA) project to investigate the change in the dynamics of a Slocum G2 UG, shown in Fig. 1, under simulated high levels of marine growth. Additionally, a new method has been developed to help pilots remotely detect the onset of marine growth based on the dynamic model of the UG in the vertical plane under steady-state conditions.

\section{Methodology}

\section{A. Deployment Description}

Slocum is a type of UG manufactured by Teledyne Webb Research that have been in operation since the late nineties [5], [6]. In this field test, the Slocum G2 UG operated by PLOCAN, as shown in Fig. 1, was employed (unit 492). The main actuator is the VBD (Fig. 2), which consists of an oil bladder that can be extended or retracted from the pressure hull. When the bladder is outside the pressure hull, the vehicle's displacement and thus its net buoyancy increase and vice versa. Pitch is controlled by shifting the position of one movable battery pack with a dedicated mechanism. Yaw is controlled through a rudder, which is magnetically coupled to a servo motor to avoid an opening in the pressure hull.

As can be seen in Fig. 2, Slocums can perform multiple "yos" per dive (two in this particular case), i.e. the vehicle can sample the water column multiple times before returning 


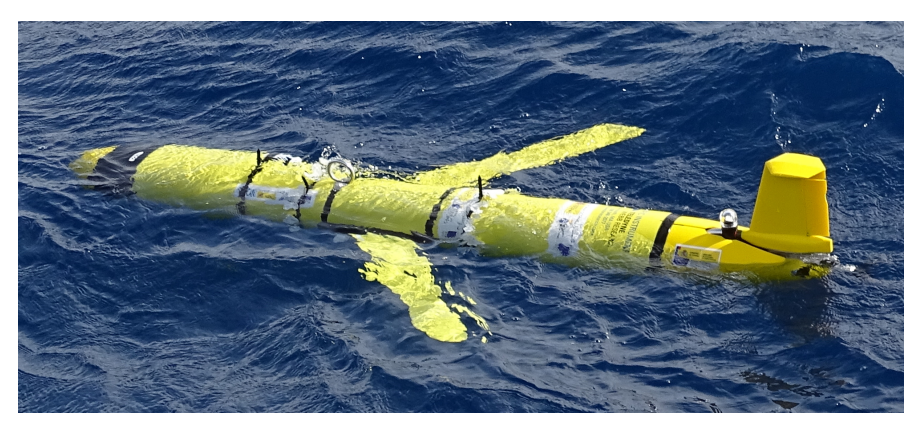

Fig. 1. Slocum G2 operated by PLOCAN.

to the surface to send and receive data by satellite and get a new position fix [7]. In particular, the minimum depth that the glider reaches between yos can be set to be lower than the surface to avoid marine traffic and biofouling [2]. The data sent ashore needs to be decimated to reduce the time that the vehicle spends on the surface, the power expenditure and the actual financial cost associated with the transmission of the data by satellite (specifically, via Iridium). The data usually includes the vehicle's orientation, its depth (from which the vertical velocity can be obtained), the actuator's signals, the capacity and voltage of the battery, the estimated location, samples of the scientific data of interest and warnings from on-board health monitoring systems. In this field test, the only scientific sensor connected to the UG's scientific bay is the Seabird Scientific CTD sensor.

The field test actually consisted of two separate missions run with the same vehicle sequentially off the coast of Gran Canaria, as shown in Fig. 3. During the first mission, the UG was deployed from $17^{\text {th }}$ to $26^{\text {th }}$ February 2020 off the east coast of the island after being covered with drag stimulators to simulate high levels of marine growth. In this test, the maximum depth was capped at $200 \mathrm{~m}$ to avoid possible structural and/or hydrostatic problems with the stimulators.

On $26^{\text {th }}$ February the vehicle was recovered, the drag stimulators removed and then the vehicle redeployed until $17^{\text {th }}$ March 2020. During this mission, the depth was increased to $1,000 \mathrm{~m}$. Whilst the scope of the first deployment was to provide a dataset of a heavily biofouled glider, the second deployment had a scientific goal. By sharing the two sets, it has been possible to have data for the same glider with both a clean and a biofouled hull.

After the first few test dives with a single yo, the number of yos per dive were set to three during both deployments. Both missions were constrained by time rather than battery energy. Hence, the full VBD capacity has been used to increase the UG's speed in the water at the expense of the higher energy cost. Thus, the magnitude of the VBD volume and pitch angle were set to $260 \mathrm{~cm}^{3}$ and $26^{\circ}$, respectively, for most dives. To increase the range of the vertical velocity and pitch angle, a few dives were run with combinations of a VBD volume of $-200 \mathrm{~cm}^{3}$ and $-260 \mathrm{~cm}^{3}$ in descents and pitch angles with a magnitude of $18^{\circ}, 26^{\circ}$ and $30^{\circ}$. In ascents, the full VBD capacity of $+260 \mathrm{~cm}^{3}$ was used at all times because of a perceived potential risk that the drag stimulators would compress with depth thus contributing to a loss of net buoyancy.

\section{B. Drag Stimulators}

As described in [2], some of the most common occurrences of severe biofouling on UGs consist of the growth of shellfish (in particular $p$. pollicipes) on the nose and at the edges of the cylindrical sections of the hull. Whilst the area around the nose presents lower flow speed due to the higher pressure associated with the front stagnation point, the junction of the cylindrical hull sections offers good grasping points for the roots of the shellfish larvae. Shallow dives, i.e. with a depth shallower than $200 \mathrm{~m}$, in warm waters have been found to especially contribute to marine growth. The main indicator of marine growth is a drop in the UG's vertical velocity for the same VBD volume and pitch angle settings.

In this study, artificial drag stimulators were designed and built to simulate high levels of marine growth as observed in [2]. In particular, a concept similar to drag stimulators to induce turbulent flow in ship model testing was envisaged [8]. Biofouling is expected to mostly impact the drag and net buoyancy of the vehicle. However, the change in net buoyancy is difficult to quantify and the UG had to be redeployed straight after the removal of the drag stimulators. Hence, to avoid serious ballasting challenges associated with two vehicle configurations, the drag stimulators have been designed to be approximately neutrally buoyant.

The final design of the drag stimulators can be seen in Fig. 4, as highlighted by the red boxes. In total, three drag stimulator belts were connected to the UG, each consisting of:

- one $780 \mathrm{~mm} \times 9 \mathrm{~mm}$ nylon cable tie,

- heat shrink cover to increase friction with the hull and prevent the ties from creeping along the hull in water; the heat shrink is split into three parts to avoid contact with the two frames of the UG's trolley,

- ten 3D-printed 40-mm-long mussels, each of which is connected to the cable tie at 50-mm intervals through high strength fishing line passing through two drilled small holes. The front drag stimulator presents ten folded, 40mm-wide neoprene strips instead of the 3D-printed shells.

TABLE I

MASS, DISPLACED VOLUME AND ORIENTATION OF THE DRAG STIMULATORS

\begin{tabular}{cccc} 
drag stimulator & mass $\mathbf{[ g ]}$ & volume $\left[\mathbf{c m}^{3}\right]$ & orientation \\
\hline Front & $52.39 \pm 0.5$ & $40 \pm 10$ & back-facing \\
Middle & $33.53 \pm 0.5$ & $25 \pm 10$ & forward-facing \\
Aft & $33.67 \pm 0.5$ & $25 \pm 10$ & back-facing
\end{tabular}

Although 3D-printed parts have been used successfully in very deep waters [9], little information could be found on the use of parts built with Selective Laser Sintering (SLS) using Polyamide 12. Hence, dives with drag stimulators were capped to a maximum depth of $200 \mathrm{~m}$ for precaution. 


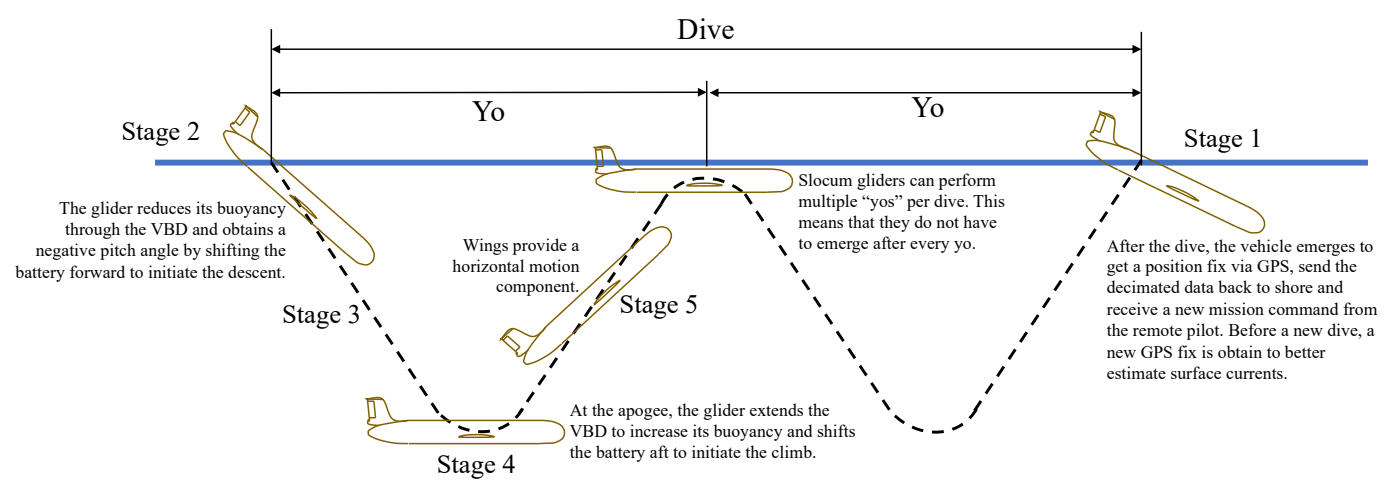

Fig. 2. Concept of operation of the UG. The drawing is not to scale: the vehicle operated in depths up to $1,000 \mathrm{~m}$ deep and with the pitch angle ranging from $15^{\circ}$ to $30^{\circ}$

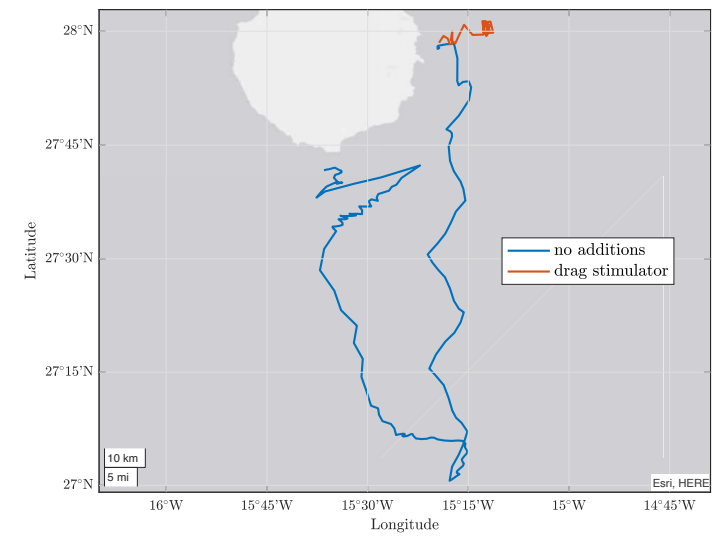

Fig. 3. Map of the two deployments.

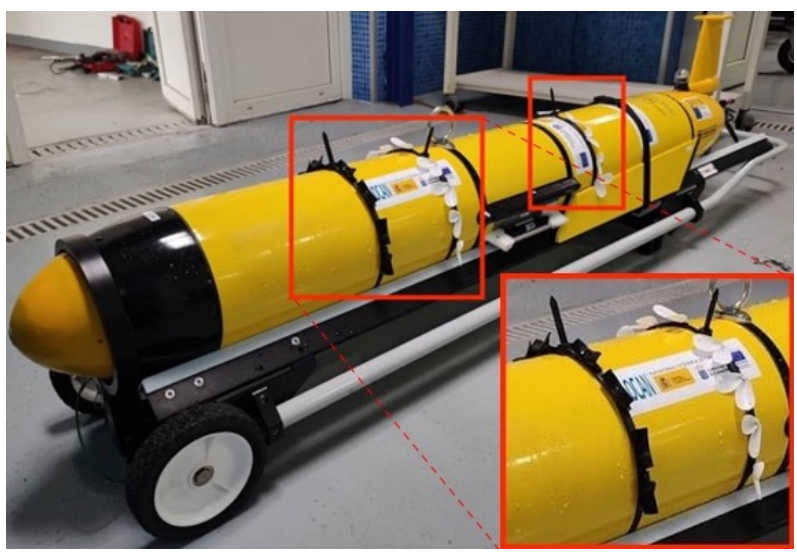

Fig. 4. Drag stimulators (highlighted by red boxes) added to the Slocum UG to simulate extreme levels of marine growth.

Table I shows the total mass and displaced volume of each drag stimulator. As can be seen, the precision of the volume measurement is much lower than for the mass measurement. However, the drag stimulators are close to neutrally buoyant in the expected water density of the deployment site (approximately $1.027 \mathrm{~g} / \mathrm{cm}^{3}$ ).

During transport of the UG to the deployment site, one 3D- a)

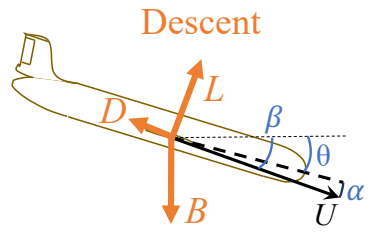

b)

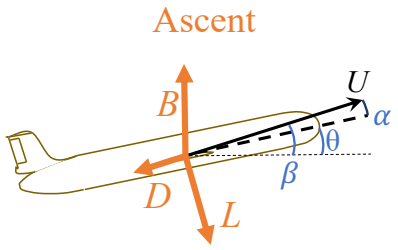

Fig. 5. Free-body diagram showing the forces acting on the UG in the vertical plane in steady-state conditions.

printed shell was lost from the middle drag stimulator. Hence, during the field test, the middle belt had only nine shells.

\section{Steady-State Dynamic Model}

The free-body diagram of the equilibrium condition for the steady-state flight is shown in Fig. 5a and Fig. 5b for descents and ascents, respectively. $B$ indicates the net buoyancy, $L$ the lift and $D$ the drag force. $U$ is the surge velocity component in the body-fixed frame, $\theta$ the pitch, $\alpha$ the attack and $\beta$ the glide-path angles. The glide-path angle indicates the angle of the flight path in the inertial reference system and is obtained from the sum of the pitch and attack angles: $\beta=\theta+\alpha$.

In both descents and ascents, the force balance yields [10]

$$
B-L \cos \beta-D \sin \beta=0 \text {. }
$$

According to the standard dynamic model for Slocum UGs in steady-state conditions [10], the drag and lift forces can be modelled as

$$
\begin{gathered}
L=\frac{1}{2} k_{\mathrm{L}} \alpha \rho S U^{2}, \\
D=\frac{1}{2}\left(k_{\mathrm{D}, 0}+k_{\mathrm{D}, \mathrm{L}} \alpha^{2}\right) \rho S U^{2},
\end{gathered}
$$


where $\rho$ is the water density, $S$ is the wetted surface area, $k_{\mathrm{L}}, k_{\mathrm{D}, 0}$ and $k_{\mathrm{D}}$ are constants used to compute the lift and drag coefficients. The water density is obtained from the water pressure, salinity and temperature using the Gibbs Seawater Toolbox [11]. These properties are measured by the CTD sensor on-board the glider.

The net buoyancy force can be computed as

$B=g\left\{-m+\rho\left[V_{0}\left(1-\epsilon_{c} p+\alpha_{T}\left(T-T_{0}\right)\right)+V_{\mathrm{vbd}}\right]\right\}+\delta B$,

where $g$ is the gravitational acceleration, $m$ is the UG mass, $V_{0}$ its reference volume, $\epsilon_{c}$ the absolute compressibility of the pressure hull and $\alpha_{T}$ its thermal expansivity, with the reference temperature $T_{0}$. The values for the parameters can be found in Table II. $p$ is the water pressure and $T$ its temperature. The offset in the net buoyancy, $\delta B$, is added over [10] to account for possible ballasting problems caused by the drag stimulators.

TABLE II

SUMMARY OF THE FIXED MODEL PARAMETERS .

\begin{tabular}{cccc} 
parameter & value & units & source \\
\hline$g$ & 9.81 & {$\left[\mathrm{~m} / \mathrm{s}^{2}\right]$} & {$[10]$} \\
$S$ & 0.1 & {$\left[\mathrm{~m}^{2}\right]$} & {$[10]$} \\
$\epsilon_{c}$ & $6.4 \times 10^{-6}$ & {$\left[\mathrm{dbar}^{-1}\right]$} & manufacturer \\
$\alpha_{T}$ & $5.3 \times 10^{-5}$ & {$\left[{ }^{\circ} \mathrm{C}^{-1}\right]$} & manufacturer \\
$m$ & 58.2428 & {$[\mathrm{~kg}]$} & ballasting \\
$V_{0}$ & 56,730 & {$\left[\mathrm{~cm}^{3}\right]$} & ballasting \\
$T_{0}$ & 19.43 & {$\left[{ }^{\circ} \mathrm{C}\right]$} & ballasting
\end{tabular}

Substituting (2), (3) and (4) into (1), it is possible to express the vertical velocity predicted by the model, $\dot{z}_{\mathrm{m}}$, as follows [10]:

$$
\begin{gathered}
\dot{z}_{\mathrm{m}}=\sqrt{U^{2}} \sin \beta, \text { where } \\
U^{2}=\frac{B \sin \beta}{0.5 \rho S\left(k_{\mathrm{D}, 0}+k_{\mathrm{D}, \mathrm{L}} \alpha^{2}\right)}, \\
\alpha=\frac{k_{\mathrm{D}, 0}+k_{\mathrm{D}, \mathrm{L}} \alpha^{2}}{k_{\mathrm{L}} \tan (\beta)} .
\end{gathered}
$$

Equation (7) requires an iterative solution. Additionally, an optimisation needs to be run to find the hydrodynamic parameters specific to the UG. The cost function is expressed as:

$$
J=\left[\frac{1}{I} \sum_{i=1}^{I}\left(\dot{z}_{i}-\dot{z}_{\mathrm{m}, i}\right)^{2} \forall i \in \mathbb{R}\right]+\left[\sum_{j=1}^{J} j \forall j \notin \mathbb{R}\right] .
$$

In (8), $i$ indicate all points for which a numeric cost value is obtained, while $j$ all points for which $U^{2}<0$. Imposing an additional cost on the number of non-numeric values speeds up convergence as compared with Merckelbach, et al. [10].

Here, a global search optimisation is run to find the desired parameters for each deployment: $k_{L}, k_{D, 0}, k_{D, L}$ and $\delta B$. A scatter search [12] is used to generate trial points within the search space from which a constrained, nonlinear programming solution is found based on a trust region method based on interior point techniques [13]. The scatter search algorithm then assesses the cost function of the solutions to update the trial points and continue the minimisation until convergence onto the global optimum. The MATLAB GlobalSearch and fmincon tools were used in the practical implementation, with default settings. The lift, drag, induced drag and buoyancy offset values were constrained to $k_{L} \in[0,25] \mathrm{rad}^{-1}, k_{D, 0} \in$ $[0,0.4], k_{D, L} \in[0,12] \mathrm{rad}^{-2}$ and $\delta B \in[-3,3] \mathrm{N}$, respectively.

\section{Power Consumption Analysis}

The impact of marine growth on the power consumption of the UG can also be analysed. In general, it is possible to identify five main operational stages that will contribute to different power consumption profiles (Fig. 2): satellite communications, actuation of the VBD and pitch control before a descent, steady-state during the descent, actuation of the VBD and pitch control before the ascent and steady-state during the ascent.

The data is cleaned to obtain the energy consumption (from the signal of the battery capacity and voltage) and duration of each stage for all dives and yos. The energy consumption and duration of the telemetry stage is a function of the amount of data that needs to be transmitted by satellite. The energy consumption and duration of the dynamic response stages is a function of the change in the actuators' settings. During the two deployments, the glider was operated at maximum VBD volume in both clean and biofouled conditions. Therefore, the most noticeable changes due to marine growth were expected in the steady-state operation. Hence, the data from the steadystate descents and ascents was merged and subjected to further statistical analysis. Since the maximum and minimum depth can have a large influence on the energy consumption and duration of each steady-state stage, only data from dives with similar maximum and minimum depths was considered (up to $200 \mathrm{~m})$.

Firstly, an analysis of covariance (ANCOVA) [14] was run to determine whether there is a significant difference in the mean power consumption during steady-state conditions between the clean and biofouled hull, whilst controlling for the effect of the mean absolute VBD volume and pitch angle during either the ascent or descent. A significance level of $99 \%$ was used.

\section{E. Remote real-time detection of marine growth levels}

During the field test, the steady-state dynamic model was applied to the decimated data sent by the UG ashore after each dive. As the data range is drastically reduced, the lift and induced drag coefficients are set to the values found in [10]. The global optimisation is thus limited to finding the drag coefficient and buoyancy offset for each dive.

\section{RESUlts AND Discussion}

\section{A. Comparison between the clean and biofouled hulls}

1) Vehicle Dynamics: As can be seen in Table III, the drag stimulators cause an increase of $92 \%$ in the drag coefficient and of $37 \%$ in the induced drag coefficient. Conversely, the impact on the net buoyancy is negligible thanks to their design. The lift coefficient is $65 \%$ lower than that observed in [10]. 


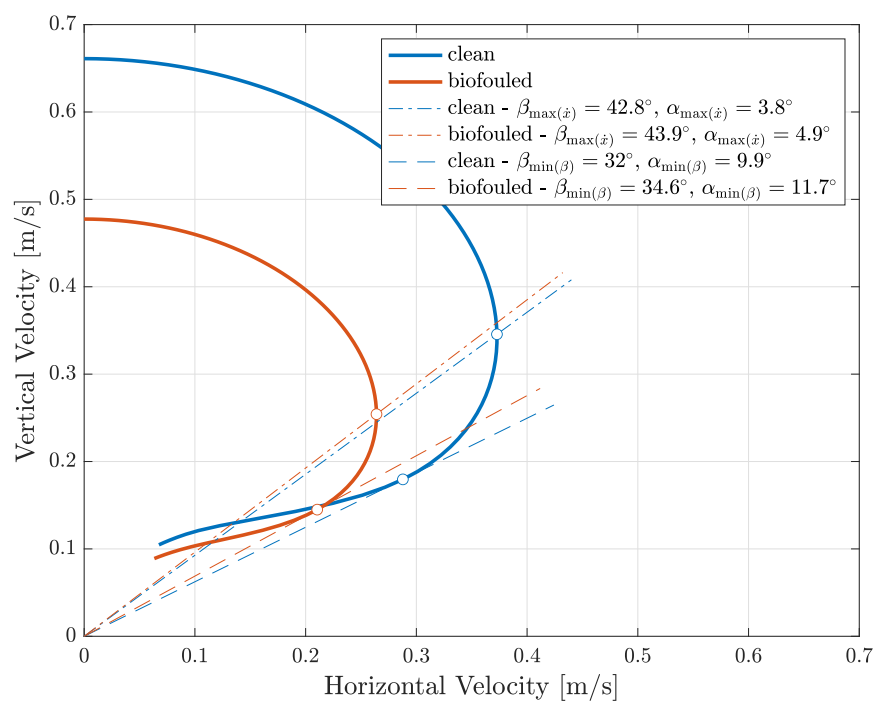

Fig. 6. glide polar plot for the UG using the parameters in Table III for the full VBD volume of $260 \mathrm{~cm}^{3}$, a water density of $1.027 \mathrm{~kg} / \mathrm{m}^{3}$ and ignoring the compressibility and thermal expansivity of the hull.

The drag stimulators increase the lift coefficient by $46 \%$. This is mainly due to the extremely large size of the 3D-printed shells of the drag stimulators. In fact, real biofouling may cause a reduction of lift coefficient [4].

TABLE III

PARAMETERS OF THE DYNAMIC MODEL FOR THE TWO HULL CONDITIONS.

\begin{tabular}{ccccc} 
Hull state & $k_{L}\left[\mathrm{rad}^{-1}\right]$ & $k_{D, 0}$ & $k_{D, L}\left[\mathrm{rad}^{-2}\right]$ & $\delta B[\mathrm{~N}]$ \\
\hline Clean & 2.659 & 0.140 & 4.759 & -0.537 \\
Biofouled & 3.881 & 0.269 & 6.525 & -0.521
\end{tabular}

The glide polar plot for the UG is shown in Fig. using the parameters in Table III for the full VBD volume of $260 \mathrm{~cm}^{3}$, a water density of $1.027 \mathrm{~kg} / \mathrm{m}^{3}$ and ignoring the compressibility and thermal expansivity of the hull. Due to the low ratio of the estimated lift and drag coefficients, the polar plot presents higher minimum glide angle and glide angle for maximum horizontal speed than theoretical predictions in [3] and [4]. The discrepancy is likely to be due to the limited range of the $\theta-\dot{z}$ search space in the analysed deployments. This justifies the reduction of the search space for the system identification to two parameters for individual dives.

2) Power Consumption Analysis: From the ANCOVA, the mean power consumption in each descent or ascent has been found to be significantly different for the clean and biofouled hull datasets $(p=0.04 \%<1 \%$ ). Additionally, the values are significantly affected by the mean VBD volume ( $p \ll 1 \%$ ), whilst the contribution of the pitch angle is not deemed significant to a $99 \%$ level ( $p=2.04 \%$ ). However, the ANCOVA results should be treated with caution, as it assumes linear relationships between the independent and continuous dependent variables and the mean square errors to be normally distributed [14].

From the charts of the energy consumption in Fig. 7,

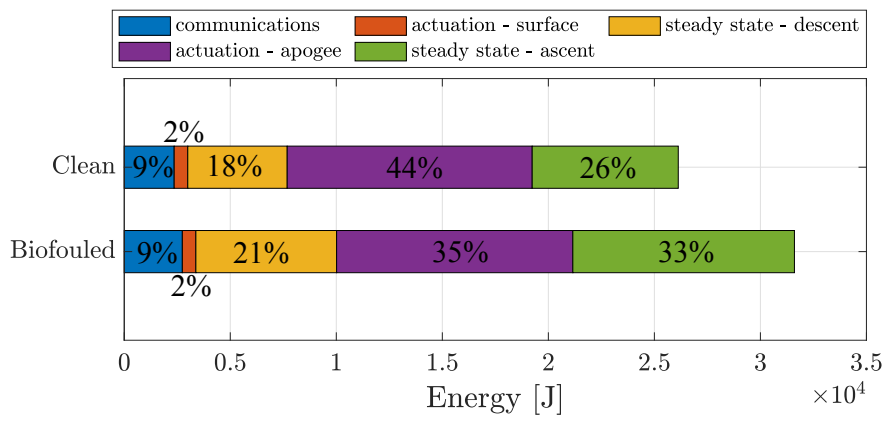

Fig. 7. Energy consumption during the identified five operational stages over a dive cycle with three yos for a $200-\mathrm{m}$ dive for the clean and biofouled UG.

it is clear the steady-state operational phases play a more important role on the overall UG's energy consumption for the biofouled hull. Hence, marine growth will reduce the deployment duration for the same available energy. This is caused by the longer duration of the steady-state phases due to the lower speed in the water.

It is interesting to note that pressure lower than atmospheric inside the pressure hull greatly helps reduce the actuation energy costs of the UG, since the VBD bladder can be retracted before descents without operating the VBD pump, which is the greatest power draw.

3) Cost of Transport: Similarly to [15], the energetic cost of transport (COT) is quantified as the ratio of the energy expenditure and the product of the vehicle's mass and distance travelled. Table IV displays the mean and standard deviation of the COT and horizontal velocity for the clean and biofouled UG in dives with a maximum depth of $200 \mathrm{~m}$ and three yos. The distance travelled during the dive is computed from the latitude and longitude at the start and end of the dive, when the UG receives a GPS fix. Similarly, the dive duration is computed from the difference of the respective time stamps. The results from the initial five set-up dives are ignored.

TABLE IV

MEAN AND STANDARD DEVIATION OF THE COT AND HORIZONTAL VELOCITY FOR A 200-M DIVE WITH THREE YOS.

\begin{tabular}{lc|cc} 
Variable & Units & Clean & Biofouled \\
\hline$\overline{\mathrm{COT}}$ & {$\left[\mathrm{J} \mathrm{m}^{-1} \mathrm{~kg}^{-1}\right]$} & 0.199 & 0.325 \\
$s_{\mathrm{COT}}$ & {$\left[\mathrm{J} \mathrm{m}^{-1} \mathrm{~kg}^{-1}\right]$} & 0.104 & 0.195 \\
$\dot{\dot{x}}$ & {$\left[\mathrm{~m} \mathrm{~s}^{-1}\right]$} & 0.344 & 0.185 \\
$s_{\dot{x}}$ & {$\left[\mathrm{~m} \mathrm{~s}^{-1}\right]$} & 0.129 & 0.067
\end{tabular}

As can be seen in Table IV, severe biofouling levels result in a rise in the COT of $63.3 \%$ and a drop in horizontal speed of $46.3 \%$. The COT results are comparable to [15], although the higher values are likely to be due to the full VBD settings used during the current deployment.

\section{B. Remote real-time detection of marine growth levels}

When recovered after the second deployment, the glider actually presented significant natural biofouling of $p$. pollicipes, as shown in Fig. 8. This was caused by the extended operation 


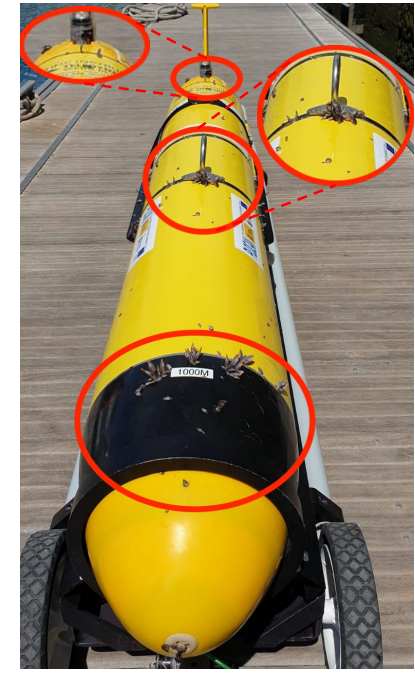

Fig. 8. Extent of actual marine growth after retrieval.

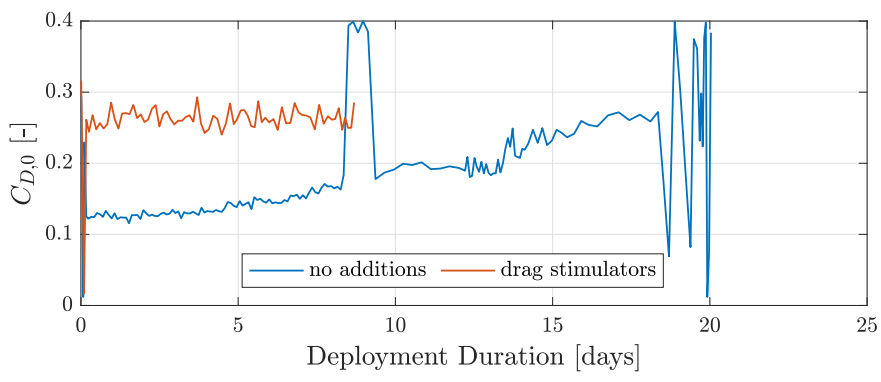

Fig. 9. Evolution of the drag coefficient with time for the two deployments.

in warm, shallow waters to simulate the event during the first deployment, which enabled the marine creatures to take root.

Figure 9 shows the time variation of the drag coefficient for the two deployments. Whilst there is no noticeable change for the first deployment, the mission without the drag stimulators presents a slowly increasing drag coefficient, which reaches levels similar to those simulated by the drag stimulators due to the actual biofouling. Hence, the developed model-based condition monitoring tool was able to track the growth of the marine creatures during the second deployment. As a result, only the data from the first six days of the second deployment was used to describe a clean hull in the previous study.

The serious oscillations for the curve for the case with no additions in Fig. 9 correspond to dives where the data were stored only for descents. Hence, the dynamic model is sensitive to the symmetry of the dive data for a correct centering.

\section{Conclusions}

High levels of marine growth, as simulated by the drag stimulators, on the UG's performance were observed to cause a drop in vertical velocity for the same VBD volume due to an increase of $92 \%$ of the drag coefficient and $37 \%$ of the induced drag coefficient. Furthermore, the share of steady-state descents and ascents increased from $26 \%$ and $18 \%$ to $33 \%$ and $21 \%$, respectively, of the overall total energy consumption for a typical three-yo dive. This is caused by the longer duration of these stages due to the lower vertical speed. As a result, high levels of marine growth will significantly reduce deployment duration for the same installed energy, with pilots needing to re-plan the mission if high levels of biofouling are detected.

A simple model-based condition monitoring tool is developed to help pilots recognise the onset of marine growth so that a safe vehicle retrieval can be planned if needed. The dynamic model is particularly affected by having data only for descents, so that navigation data from both descents and ascents should be sent by satellite after decimation if modelbased diagnostics are to be used. In the future, data-driven fault diagnostics tools may be required to address this challenge.

\section{REFERENCES}

[1] D. L. Rudnick, "Ocean Research Enabled by Underwater Gliders," Annual Review of Marine Science, vol. 8, no. 1, pp. 519-541, 2016.

[2] C. D. I. Haldeman, D. K. Aragon, T. Miles, Scott M. Glenn, and A. G. Ramos, "Lessening biofouling on long-duration AUV flights: Behavior modifications and lessons learned," in MTS/IEEE Oceans. Monterey, California, USA: MTS/IEEE, 2016.

[3] M. Eichhorn, D. Aragon, Y. A. Shardt, and H. Roarty, "Modeling for the performance of navigation, control and data post-processing of underwater gliders," Applied Ocean Research, vol. 101, p. 102191, aug 2020.

[4] C. Deutsch, J. Kuttenkeuler, and T. Melin, "Glider performance analysis and intermediate-fidelity modelling of underwater vehicles," Ocean Engineering, vol. 210, aug 2020.

[5] D. C. Webb, P. J. Simonetti, and C. P. Jones, "SLOCUM: An underwater glider propelled by environmental energy," IEEE Journal of Oceanic Engineering, 2001.

[6] O. Schofield, J. Kohut, D. Aragon, L. Creed, J. Graver, C. Haldeman, J. Kerfoot, H. Roarty, C. Jones, D. Webb, and S. Glenn, "Slocum Gliders: Robust and ready," Journal of Field Robotics, vol. 24, no. 6, pp. 474485, 2007.

[7] Teledyne Webb Research, "Slocum G2 Glider Operators Manual," Teledyne Webb Research, Tech. Rep., 2012. [Online]. Available: www.webbresearch.com

[8] T. C. Fu, H. Tanaka, J. Kim, G. Macfarlane, W. Wang, R. Pattenden, M. Felli, and S. Bal, "The Resistance Committee: Final Report and Recommendations to the 28th ITTC," in Proceedings of the 28th ITTC, Volume I, Wuxi, China, 2017.

[9] D. M. Vogt, K. P. Becker, B. T. Phillips, M. A. Graule, R. D. Rotjan, T. M. Shank, E. E. Cordes, R. J. Wood, and D. F. Gruber, "Shipboard design and fabrication of custom 3D-printed soft robotic manipulators for the investigation of delicate deep-sea organisms," PLoS ONE, vol. 13, no. 8, aug 2018 .

[10] L. Merckelbach, A. Berger, G. Krahmann, M. Dengler, and J. R. Carpenter, "A dynamic flight model for Slocum gliders and implications for turbulence microstructure measurements," Journal of Atmospheric and Oceanic Technology, vol. 36, pp. 281-296, jan 2019.

[11] T. McDougall and P. Barker, "Getting started with TEOS-10 and the Gibbs Seawater (GSW) Oceanographic Toolbox," SCOR/IAPSO WG 127, p. 28, 2011.

[12] Z. Ugray, L. Lasdon, J. Plummer, and F. Glover, "Scatter Search and Local NLP Solvers : A Multistart Framework for Global Optimization," Information Systems, vol. 19, no. 3, pp. 328-340, 2007.

[13] R. H. Byrd, J. C. Gilbert, and J. Nocedal, "A trust region method based on interior point techniques for nonlinear programming," Mathematical Programming, Series B, vol. 89, no. 1, pp. 149-185, 2000.

[14] T. Hastie, R. Tibshirani, and J. Friedman, Elements Of Statistical Learning Data, 2nd ed. Springer Verlag, 2009.

[15] A. B. Phillips, M. Haroutunian, S. K. Man, A. J. Murphy, S. W. Boyd, J. I. R. Blake, and G. Griffiths, "Nature in Engineering for Monitoring the Oceans: Comparison of the energetic costs of marine animals and AUVs," in Further Advances in Unmanned Marine Vehicles, G. Roberts and R. Sutton, Eds. IET Control Engineering Series, 2012, pp. 373-405. 